\title{
Isoliquiritigenin Inhibits Cigarette Smoke-Induced COPD by Attenuating Inflammation and Oxidative Stress via the Regulation of the Nrf2 and NF-kB Signaling Pathways
}

\author{
Duo Yu ${ }^{1}$, Xueshibojie Liu' ${ }^{2}$, Guangxin Zhang ${ }^{3}$, Zhihui Ming ${ }^{4 *}$ and Tiejun Wang ${ }^{1 *}$ \\ ${ }^{1}$ Department of Radiotherapy, The Second Affiliated Hospital of Jilin University, Changchun, China, ${ }^{2}$ Department of Head \\ and Neck Surgery, The Second Affiliated Hospital of Jilin University, Changchun, China, ${ }^{3}$ Department of Thoracic Surgery, \\ The Second Affiliated Hospital of Jilin University, Changchun, China, ${ }^{4}$ Department of Stomatology, The Second Affiliated \\ Hospital of Jilin University, Changchun, China
}

OPEN ACCESS

Edited by:

Chiara Bolego,

University of Padova, Italy

Reviewed by:

Claudio Ferrante, Università degli Studi G. d'Annunzio

Chieti e Pescara, Italy

Lucia Recinella,

Università degli Studi G. d'Annunzio

Chieti e Pescara, Italy

*Correspondence:

Zhihui Ming

mingzhihui11@sina.com

Tiejun Wang

wangtiejunlx@sina.com

Specialty section:

This article was submitted to Inflammation Pharmacology,

a section of the journal

Frontiers in Pharmacology

Received: 25 April 2018

Accepted: 14 August 2018

Published: 20 September 2018

Citation:

Yu D, Liu X, Zhang G, Ming Z and Wang $T$ (2018) Isoliquiritigenin Inhibits Cigarette Smoke-Induced COPD by Attenuating Inflammation and Oxidative Stress via the Regulation of the Nrf2 and NF- $\kappa B$ Signaling Pathways.

Front. Pharmacol. 9:1001. doi: 10.3389/fphar.2018.01001
Chronic obstructive pulmonary disease (COPD) is the major leading cause of disease with high-mortality worldwide. Cigarette smoke (CS) is a major factor for COPD. CS causes chronic inflammation and oxidative stress, which contributes to lung dysfunction in COPD. Isoliquiritigenin (ILG), a natural flavonoid derived from the root of liquorice, has been reported to possess antiinflammatory and antioxidant activity. In the present study, we tested the mechanism and protective effects of ILG on CS-induced COPD. Mice were exposed to CS for $2 \mathrm{~h}$ twice a day for 4 weeks. ILG was given orally $1 \mathrm{~h}$ before CS exposure twice a day for 4 weeks. The bronchial alveolar lavage fluid was collected to test the levels of inflammatory cytokines and the number of inflammatory cells. The lung tissues were obtained to evaluate the pathological changes, lung edema, myeloperoxidase (MPO) activity, malondialdehyde (MDA) level, as well as the expression of the nuclear factor-erythroid 2 (Nrf2) and nuclear factor $\kappa B(N F-\kappa B)$ signaling pathways. The results showed that ILG reduced the infiltration of inflammatory cells and the production of inflammatory cytokines. ILG also reversed CS-induced lung pathological injuries, wet/dry ratio, MPO activity, and MDA level. Further research also showed that ILG dose-dependently up-regulated the expression of Nrf2 and downregulated the expression of NF-kB signaling pathways induced by CS. In conclusion, ILG protected against CS-induced COPD by inhibiting inflammatory and oxidative stress via the regulation of the Nrf2 and NF-кB signaling pathways.

Keywords: cigarette smoke, lung inflammation, Nrf2, NF-кB, cytokines

\section{INTRODUCTION}

Chronic obstructive pulmonary disease (COPD) is a highly prevalent disease that is characterized by persistent respiratory symptoms due to small airway wall fibrosis, thickening, hypersecretion of mucus, oxidative stress, and inflammation in the lung (Zheng et al., 2008; Eapen et al., 2017; Vogelmeier et al., 2017a). Evidence indicates that COPD is the fourth leading cause of death in 
the world and is projected to become the third leading cause of death by 2020 (Lykkegaard et al., 2013; Li et al., 2017). Cigarette smoke (CS) is the most important risk factor for COPD (Du et al., 2017). Exposure to CS leads to the pathogenesis of COPD through increasing oxidative stress and inflammation in the lung (Chen et al., 2010; Du et al., 2017). Hence, it is necessary to develop new drugs with antioxidant and anti-inflammatory properties that also efficiently show down the progression of COPD.

Isoliquiritigenin (ILG), a natural flavonoid derived from the root of liquorice, has been widely used as the traditional Chinese medicine in western counties (Wu et al., 2016). ILG has various benefits, including antioxidant, anticancer, antiinflammation, and anti-apoptotic properties (Traboulsi et al., 2015; Gao et al., 2017; Hou et al., 2017). Studies have demonstrated that ILG inhibits early brain impairments by regulating nuclear factor $\kappa \mathrm{B}(\mathrm{NF}-\kappa \mathrm{B})$ and NLRP3 inflammasome pathways via triggering nuclear factor-erythroid 2 (Nrf2) activity (Zeng et al., 2017). Additionally, this inhibition of the NF-кB and NLRP3 inflammasome is an anti-inflammatory mechanism common to other flavonoids (Li et al., 2016; Menghini et al., 2016). ILG reduced the protein levels of Bax, cleaved-caspase3 , cleaved-caspase-9, and increased the level of anti-apoptotic $\mathrm{Bcl}-2$ induced by IL-1 $\beta$. ILG also inhibited NF- $\kappa \mathrm{B}$ activation induced by IL-1 $\beta$ in chondrocyte-like ATDC5 cells (Ji et al., 2017). ILG has previously been shown to inhibit adipose tissue inflammation by inhibiting both NLRP3 inflammasomedependent and independent mechanisms and reducing the adipose tissue fibrosis by regulating sensors of innate immunity (Watanabe et al., 2016). However, it remains unclear whether ILG is effective for treating COPD. Thus, the mechanism and protective effect of ILG on CS-induced COPD were tested in the present study.

\section{MATERIALS AND METHODS}

\section{Animals}

Sixty C57BL/6N male mice, 6-8 weeks, were purchased from Experimental Animal Center of Baiqiuen Medical College of Jilin University. The mice were housed under pathogen-free conditions with sufficient water and food and subjected to a lightdark cycle of $12 \mathrm{~h}$. All experimental procedures complied with the requirements of the Animal Ethics Committee of Jilin University (Permit Number: 20170612). All procedures were approved by the Guide for the Care and the Use of Laboratory Animals manual published by the US National Institutes of Health.

\section{Materials}

Isoliquiritigenin was purchased from Sigma (Signma-Aldrich, United States). Cigarettes were purchased from Tobacco Group Company Limited. Myeloperoxidase (MPO) and malondialdehyde (MDA) assay kits were purchased from Jiancheng Bioengineering Institute (Nanjing, China). TNF- $\alpha$ and IL-1 $\beta$ ELISA kits were purchased from eBioscience (San Diego,

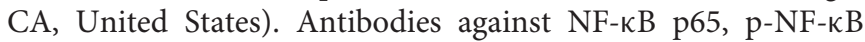
$\mathrm{p} 65, \mathrm{I} \kappa \mathrm{B} \alpha, \mathrm{p}$-I $\mathrm{B} \alpha, \mathrm{Nrf} 2$, and $\beta$-actin were purchased from Cell
Signaling Technology Inc., (Beverly, MA, United States). All other chemicals were of reagent grade.

\section{Animal Model Administration and Treatment}

Sixty mice were randomly divided into five groups and each group contained 12 mice, including the control group, the CS group, the ILG $(10,20$, and $30 \mathrm{mg} / \mathrm{kg})+$ CS groups. The CSinduced COPD model was established as described previously (Luo et al., 2017). Mice were exposed to CS for $2 \mathrm{~h}$ twice a day for 4 weeks. The control group was exposed to room air. Mice from the ILG + CS groups were given 10, 20, and $30 \mathrm{mg} / \mathrm{kg}$ ILG (diluted in PBS) orally $1 \mathrm{~h}$ before CS administration twice a day for 4 weeks. The doses of ILG used in this study were based on a previous study (Liu et al., 2017). Mice from the control group were treated with the same volume of sterile PBS. Mice were sacrificed 4 weeks later. Bronchial alveolar lavage fluid (BALF) and lung tissues were collected tor testing.

\section{BALF Collection}

The BALF was collected as previously described (Xueshibojie et al., 2016). After the last exposure of CS, mice were exsanguinated, and the thorax was opened. Five hundred microliter of PBS was injected into, retrieved from the trachea, and repeated three times. The samples were centrifuged at $3000 \mathrm{rpm}$ for $10 \mathrm{~min}$, and the supernatant was stored at $-80^{\circ} \mathrm{C}$ until use.

\section{Lung Pathological Evaluation}

The lung tissues that were not subjected to BALF were used to evaluate pathological changes. The samples were fixed in 10\% formalin for $48 \mathrm{~h}$ and dehydrated in a series of graded ethanol, embedded in paraffin, cut into $4 \mu \mathrm{m}$ sections. The section was stained with haematoxylin and eosin for pathological assay.

\section{Lung Wet/Dry Ratio Assay}

The lung wet/dry ratio was used as an index of lung edema. The upper lobe of the right lung was immediately weighed after collection and recorded as wet weight. The lung tissues were then placed into an oven at $60^{\circ} \mathrm{C}$ for $72 \mathrm{~h}$ and weighed as dry weight. The lung wet/dry ratio was calculated by dividing the wet by the dry weights.

\section{MPO and MDA Assay}

Mice were sacrificed, and the lung tissues were collected. The samples were homogenized and dissolved in extraction buffer. To test the number of neutrophils and the level of lipid peroxidation in the lung tissues, MPO and MDA levels were tested by MPO and MDA detection kits in accordance with the manufacturer's protocols.

\section{Inflammatory Cytokines Assay in BALF}

The levels of inflammatory cytokines TNF- $\alpha$ and IL- $1 \beta$ in BALF were tested by TNF- $\alpha$ and IL- $1 \beta$ ELISA kits according to the manufacturer's instruction (Xueshibojie et al., 2016). The standard curve was established according to the standards 


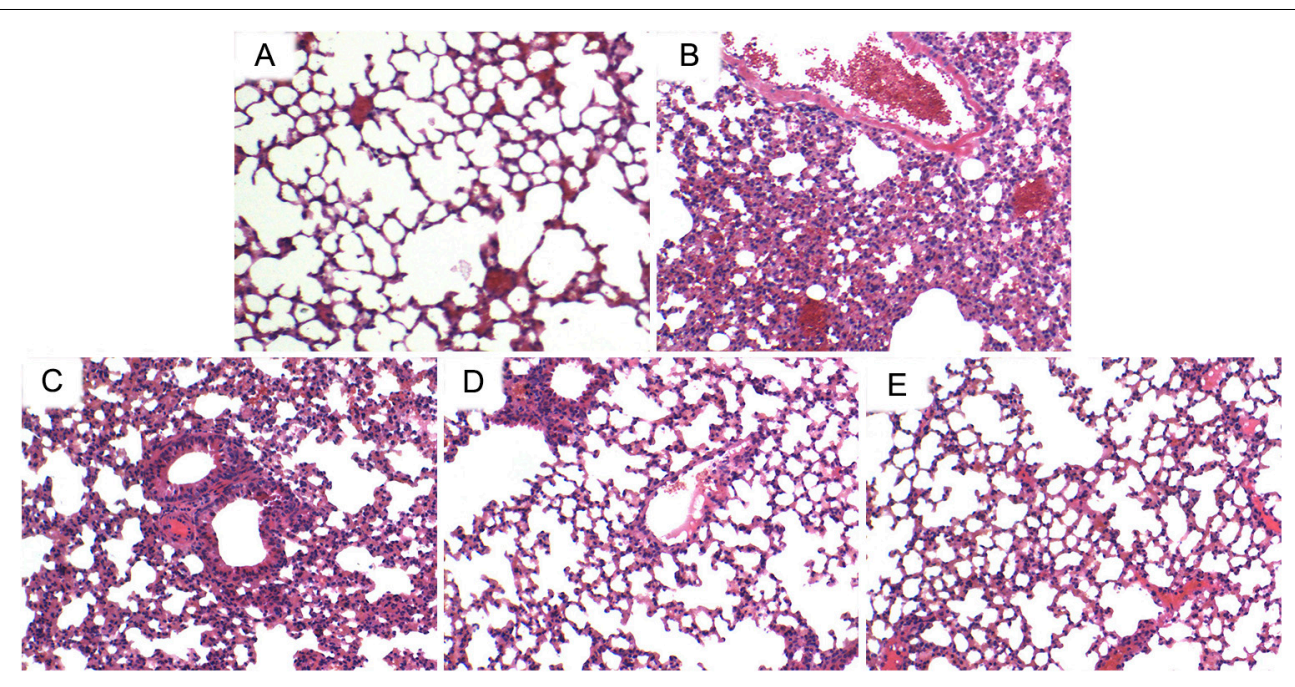

FIGURE 1 | Effects of isoliquiritigenin (ILG) on CS-induced histological changes in lung tissues. Representative histological changes of lung obtained from mice of different groups. (A) Control group, (B) CS group, (C) CS+ ILG (10 mg/kg) group, (D) CS + ILG (20 mg/kg) group, (E) CS + ILG (30 mg/kg) group (hematoxylin and eosin staining, magnification 200x).

provided by the kits. The sensitivity for TNF- $\alpha$ and IL-1 $\beta$ ELISA kits were 31.3 and $25 \mathrm{pg} / \mathrm{mL}$, respectively. Inter-assay and Intra-assay coefficients of variation were $4.1-8.3 \%$ and $2.7-8.3 \%$, respectively.

\section{Inflammatory Cell Count Assay in BALF}

The numbers of total cells, neutrophils, and macrophages in the BALF were tested in this study as previously described (Xueshibojie et al., 2016). Briefly, BALF was collected and centrifuged at $3000 \mathrm{rpm}$ at $4^{\circ} \mathrm{C}$ for $10 \mathrm{~min}$. The cell pellets were resuspended in $0.2 \mathrm{~mL}$ PBS, and the total cell counts were performed using a hemocytometer. For differential cells counts, cytospin slides were prepared, stained with Wright's stain, and 300 cells were counted per slide.

\section{Western Blot Assay}

The total protein in the lung tissues were analyzed by Western blot as previously described (Cheng et al., 2015). The protein concentration was tested by BCA kit. The protein was separated in $12 \%$ Tris-glycine SDS polyacrylamide gel and transferred onto polyvinylidene fluoride membranes. After incubation for $2 \mathrm{~h}$ with $3 \% \mathrm{BSA}$ at room temperature, the membranes were incubated with anti-NF- $\mathrm{KB}$ p65, anti-NF-

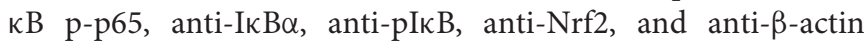
antibodies at $4^{\circ} \mathrm{C}$ overnight. After washing three times, the membranes were incubated with secondary antibody at room temperature for $45 \mathrm{~min}$. The membranes were washed three times again, and the membranes were developed with an enhanced chemiluminescence Western blotting detection system.

\section{Statistical Analysis}

Data were analyzed using the SPSS statistical software, version 17.0. Values were expressed as the mean \pm standard deviation (SD) of three independent experiments. Oneway analysis of variance (ANOVA) followed by Tukey's post hoc method was used for multiple comparisons among groups. A $p$-value of less than 0.05 was considered statistically significant.

\section{RESULTS}

\section{ILG Prevents CS-Induced Lung Pathologic Changes}

$\mathrm{H} \& \mathrm{E}$ staining was used to assess the pathological changes of the lung tissues. The results indicated that the lung tissues from CS group showed markedly pathological changes, including a large number of infiltrating inflammatory cells, and intraalveolar edema compared with lung tissues from the control group. However, administration of ILG significantly reduced these changes induced by CS (Figure 1).

\section{ILG Attenuates CS-Induced Lung Wet/Dry Ratio}

To evaluate the severity of lung edema, the lung wet/dry ratio was tested in the present study. The results showed that lung wet/dry ratio was obviously increased after CS exposure. Furthermore, the lung wet/dry ratio was attenuated by ILG in a dose-dependent manner (Figure 2).

\section{ILG Attenuates CS-Induced MPO Activity in the Lung}

MPO activity was used to test the accumulation of neutrophils in the lung tissues. As shown in Figure 3, MPO activity was significantly increased after CS exposure. And MPO activity markedly reduced after ILG treatment in a dose-dependent manner. 


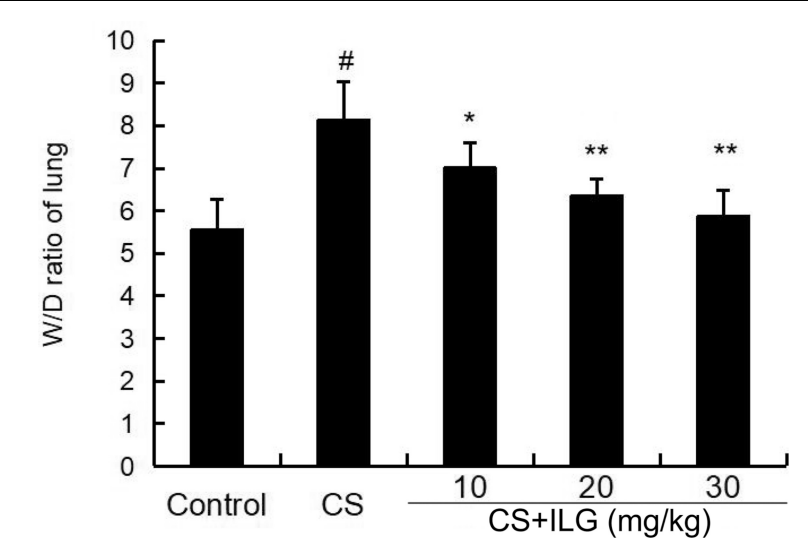

FIGURE 2 | Effects of isoliquiritigenin on lung edema of cigarette smoke (CS)-induced lung inflammation in mice. The values presented are the means \pm SEM of three independent experiments. \#p $<0.01$ vs. control group, ${ }^{*} p<0.05,{ }^{* *} p<0.01$ vs. CS group.

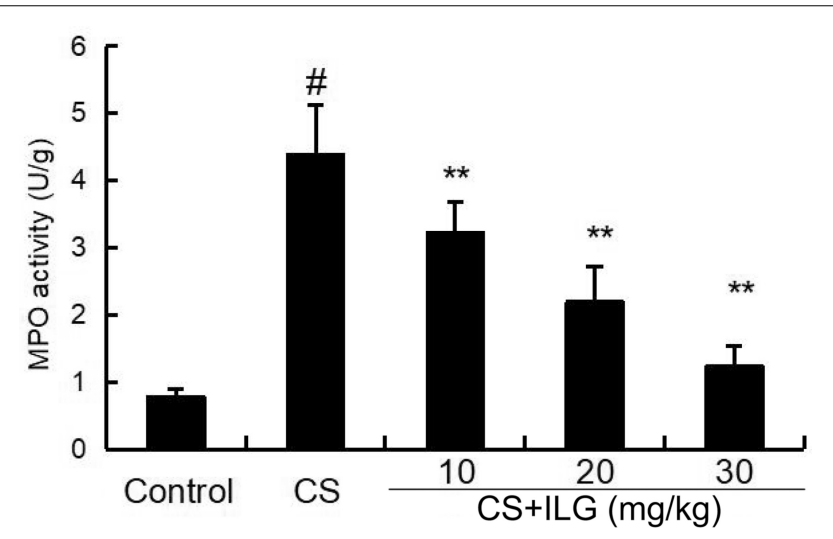

FIGURE 3 | Effects of isoliquiritigenin on myeloperoxidase activity of cigarette smoke (CS)-induced lung inflammation in mice. Lung tissues were collected at $24 \mathrm{~h}$ after the last CS exposure. The values presented are the means \pm SEM of three independent experiments. \#p $<0.01$ vs. control group, ${ }^{* *} p<0.01$ vs. CS group.

\section{ILG Attenuates CS-Induced MDA Level in the Lung}

As shown in Figure 4, increased levels of MDA were found in the lung tissues from the CS group compared with the lung tissues from the control group. Compared to the CS group, ILG cotreatment reduced CS-induced lipid peroxidation as indicated by inhibiting the level of MDA in the lung tissues.

\section{ILG Attenuates CS-Induced Inflammatory Cytokines Levels in BALF}

The effects of ILG after CS exposure on the levels of inflammatory cytokines TNF- $\alpha$ and IL- $1 \beta$ levels were analyzed by ELISA. The results showed that the levels of TNF- $\alpha$ and IL- $1 \beta$ in the BALF were significantly increased in the CS group compared with those in the control group. However, treatment with ILG reversed the levels of TNF- $\alpha$ and IL-1 $\beta$ induced by CS (Figure 5).

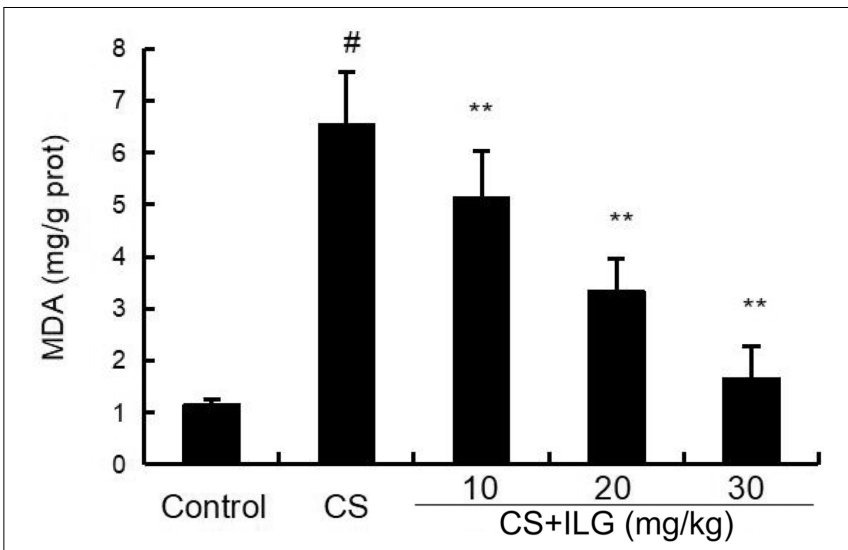

FIGURE 4 | Effects of isoliquiritigenin on malondialdehyde production of cigarette smoke (CS)-induced lung inflammation in mice. Bronchial alveolar lavage fluid was collected at $24 \mathrm{~h}$ after the last CS exposure. The values presented are the means \pm SEM of three independent experiments. $\# p<0.01$ vs. control group, ${ }^{* *} p<0.01$ vs. CS group.

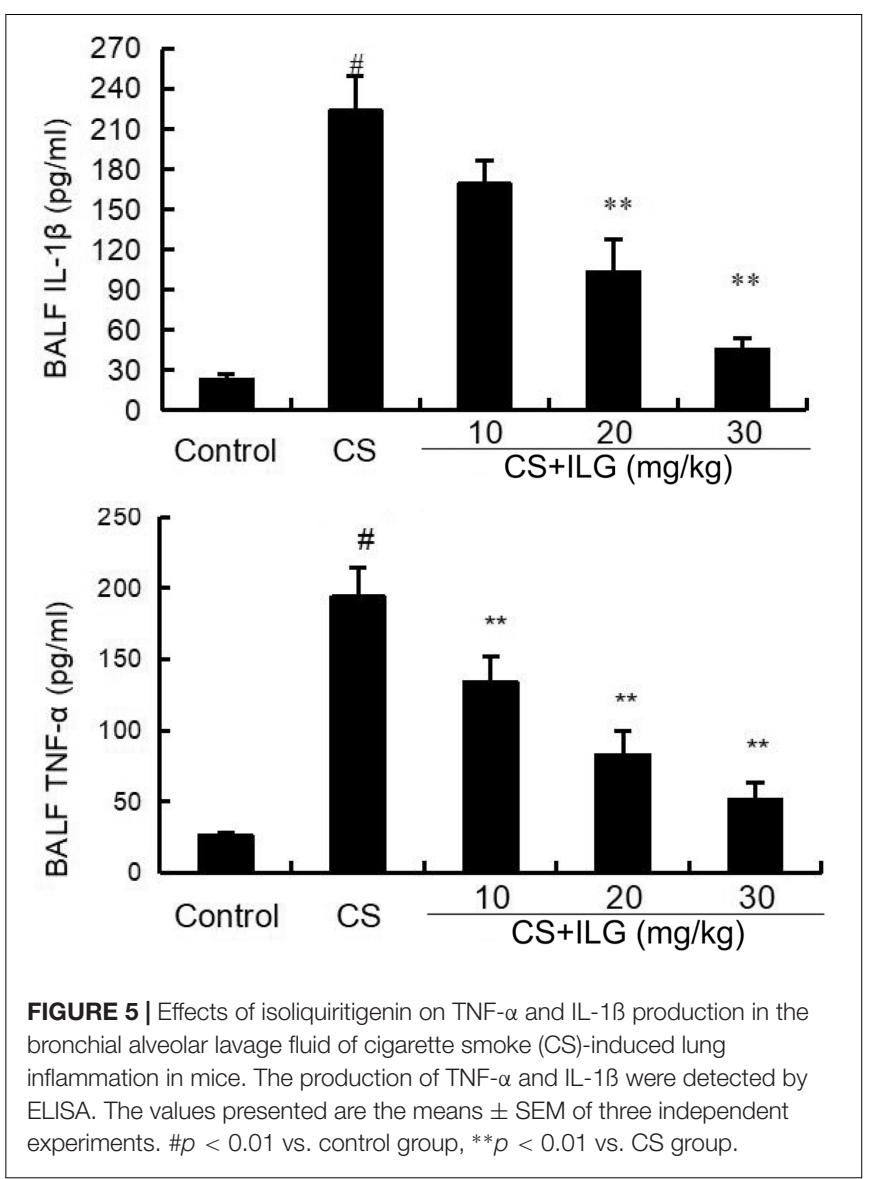

\section{ILG Attenuates CS-Induced Inflammatory Cell Counts in the BALF}

The total number of inflammatory cells, neutrophils, and macrophages in the BALF were calculated after CS exposure. The results showed that CS exposure markedly increased the number 


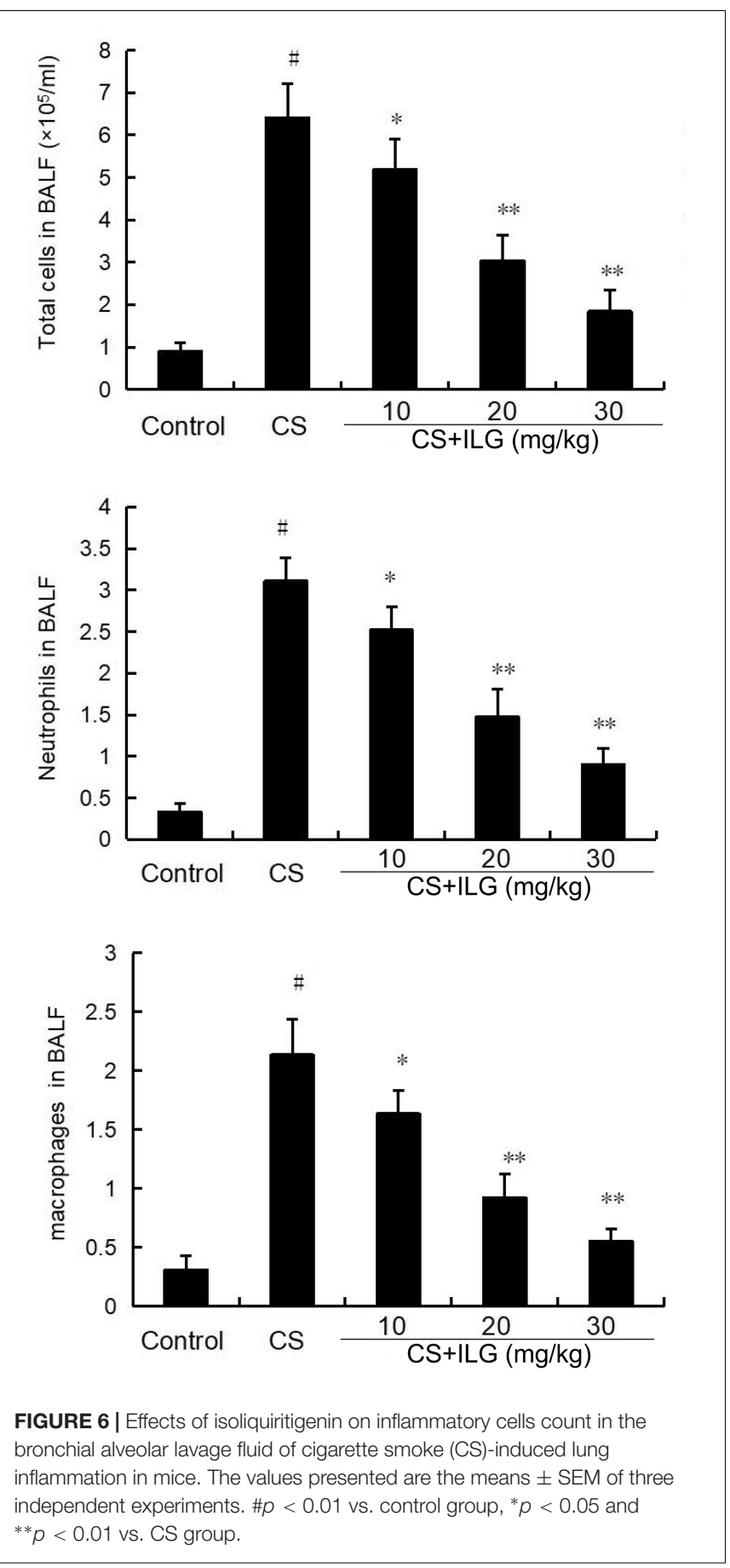

of total cells, neutrophils, and macrophages compared the control group. ILG treatment at the doses of 10,20 , and $30 \mathrm{mg} / \mathrm{kg}$ markedly reduced the number of total cells, neutrophils, and macrophages (Figure 6).

\section{ILG Attenuates CS-Induced NF-кB Activation in the Lung}

The effects of ILG on CS-induced NF- $\mathrm{B}$ activation was tested by Western blot analysis. The results showed that

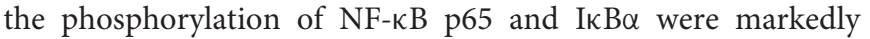
increased in the CS group compared with the control group. Treatment of ILG dose-dependently reduced the expression of

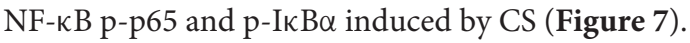

\section{ILG Upregulates CS-Induced Nrf2 Activation in the Lung}

To study the anti-oxidant effects of ILG, the protein expression of Nrf2 and HO-1 were tested by Western blot. As shown in Figure 8, the levels of Nrf2 and HO-1 were up-regulated after $\mathrm{CS}$ exposure. This up-regulation of $\mathrm{Nfr} 2$ and $\mathrm{HO}-1$ were further increased by ILG in a dose-dependent manner.

\section{DISCUSSION}

Chronic obstructive pulmonary disease is a life-threatening syndrome associated with high morbidity and mortality rates around the world (Cheng et al., 2015; Vogelmeier et al., 2017b). The major characteristics of COPD are chronic inflammation in the lung that results in reduced airflow. CS is thought to be a key factor that contributes to COPD. CS is an important inducer of oxidative stress and the inflammatory response, which leads to the pathogenesis of COPD (van der Vaart et al., 2004; Churg et al., 2008; Cheng et al., 2015). ILG, extracted from the root of liquorice, has been widely used to cure many inflammatory diseases, because of its anticancer, antioxidant, and anti-inflammatory properties (Zeng et al., 2017; Chen et al., 2018; Li et al., 2018; Na et al., 2018). In the present study, we tested the mechanism and protective effects of ILG on CS-induced COPD. The results showed that in the lung, ILG dose-dependently inhibited pathological damage, wet/dry ratio, MPO activity, MDA level, production of inflammatory cytokines, and number of inflammatory cells in BALF induced by CS. To test the mechanism of ILG on CS-induced COPD, activation of the Nrf2 and NF- $\kappa \mathrm{B}$ signaling pathways were tested. The results showed that treatment of ILG significantly inhibited the activation of NF- $\mathrm{KB}$ and promoted the activation of Nrf2 induced by CS. These data demonstrated that ILG protected against CS-induced COPD by inhibiting inflammation and oxidative stress via the regulation of $\mathrm{Nrf2}$ and NF- $\mathrm{kB}$ signaling pathway activation.

Inflammatory cytokines TNF- $\alpha$ and IL- $1 \beta$ play an important role in COPD. Over-production of TNF- $\alpha$ and IL- $1 \beta$ could amplify the inflammatory response and lead to lung dysfunction (Churg et al., 2002; Cheng et al., 2015). In addition, these cytokines can induce the recruitment of neutrophils to the lung, causing chronic bronchial inflammation and emphysema (Churg et al., 2002; Cheng et al., 2015). Our results showed that ILG significantly inhibited the levels of inflammatory cytokines TNF- $\alpha$ and IL- $1 \beta$ induced by CS. Increased MPO activity is a major indicator of neutrophils (Shen et al., 2014; Shin et al., 2016). In the present study, increased MPO activity was found in the CS-treated groups. And treatment with ILG markedly reduced MPO activity induced by CS. To test the effects of ILG on lung edema, the lung wet/dry ratio was measured in the present study. ILG dose-dependently 


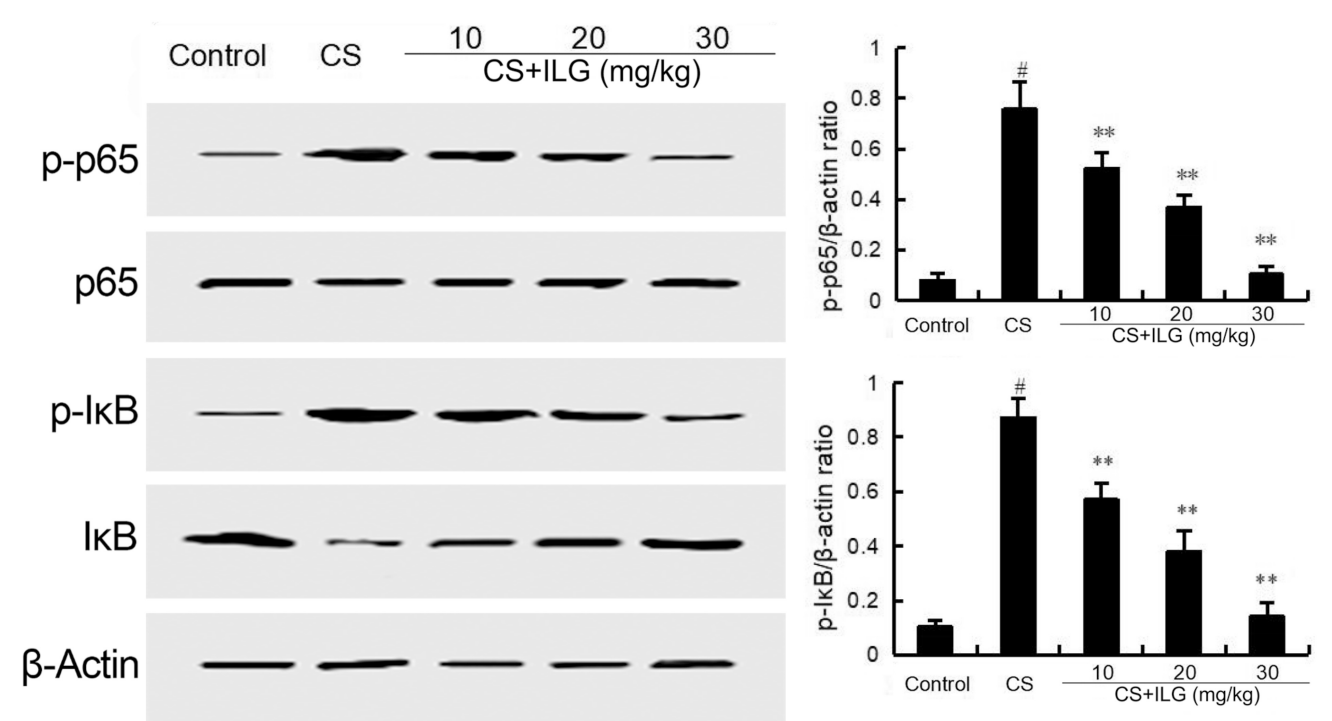

FIGURE 7 | Isoliquiritigenin inhibits cigarette smoke (CS)-induced NF-kB activation in lung tissues. The values presented are the means \pm SEM of three independent experiments. $\# p<0.01$ vs. control group, ${ }^{* *} p<0.01$ vs. CS group.

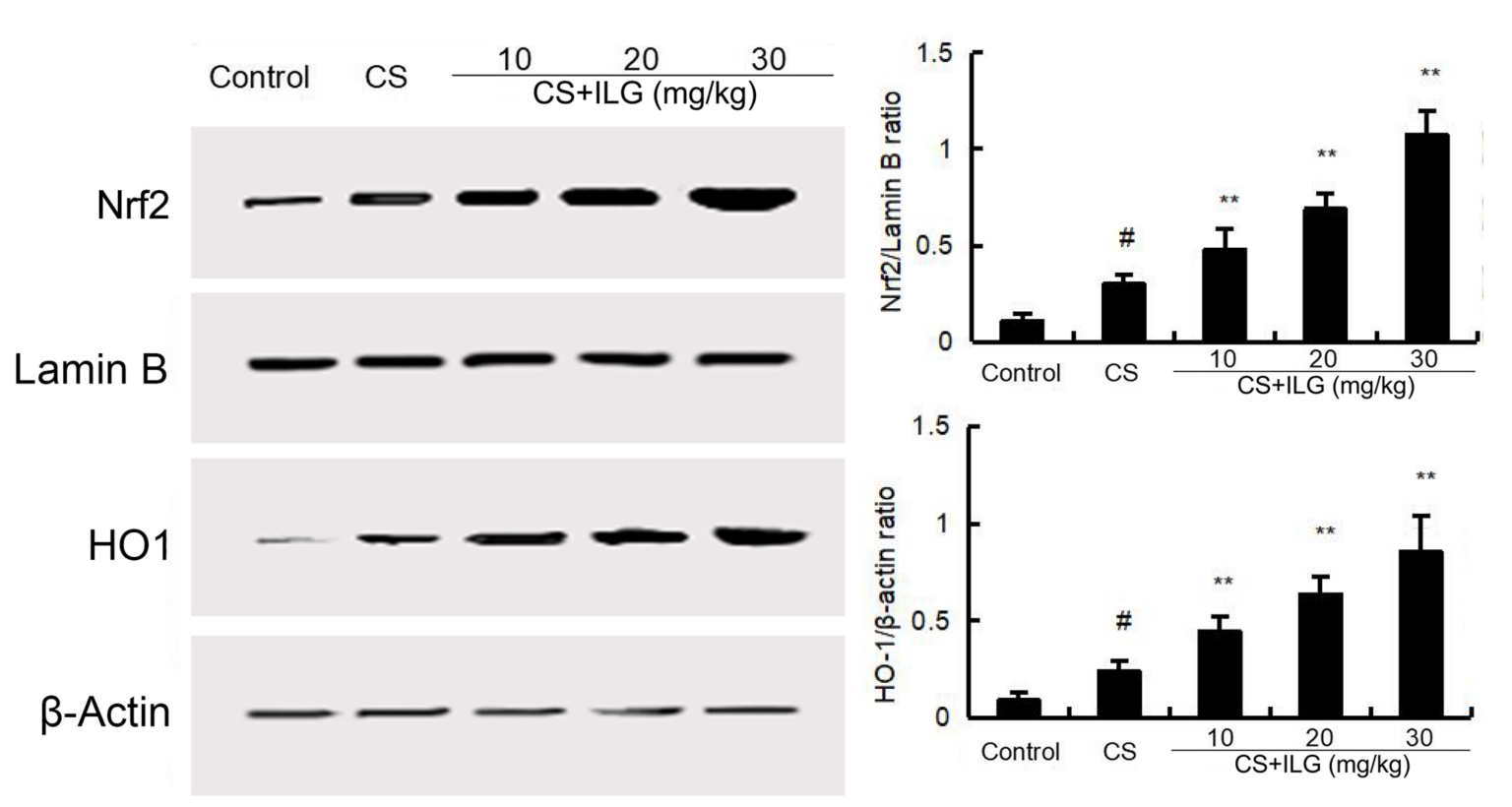

FIGURE 8 | Effects of isoliquiritigenin on Nrf2 and HO-1 expression in lung tissues. The values presented are the means \pm SEM of three independent experiments. $\# p<0.01$ vs. control group, ${ }^{* *} p<0.01$ vs. cigarette smoke group.

reduced lung wet/dry ratio induced by CS. In addition, inflammatory cells, including neutrophils, and macrophages play an important role in the inflammatory response (Moffet et al., 2016; Xu et al., 2016). In the present study, we found that total number of cells, neutrophils, and macrophages were significantly increased in the BALF from the CS groups compared with the control group. Administration of ILG reduced the total number of cells, neutrophils, and macrophages induced by $\mathrm{CS}$ in a dose-dependent manner. These data proved that ILG has an ability to protect lungs from CS-induced COPD.

Neutrophils and macrophages are the major inflammatory cells that generate ROS and inflammatory mediators that accelerate injury in the lung (Forman and Torres, 2002). Oxidative stress also plays a critical role in the development of COPD (Zuo et al., 2014). Increased lipid peroxidation was observed in mice of CS-induced COPD, and the increased lipid peroxidation could also induce lung injury 
(Thayaparan et al., 2016). In this study, we assessed the lipid peroxidation by measuring MDA content. Our results showed that ILG significantly inhibited CS-induced MDA production. Nrf2 is an important transcription factor that is involved in regulating the antioxidant response. Evidence suggests that the expression of $\mathrm{Nrf} 2$ play an important role in CS-induced COPD (Rangasamy et al., 2004; Singh et al., 2009). Up-regulation of Nrf2 improved CS-induced oxidant stress in rat lungs. In addition, studies have reported that NF$\kappa \mathrm{B}$ activation is associated with the development of COPD. Activation of Nrf2 protect against CS-induced lung inflammation by regulating NF- $\kappa \mathrm{B}$ activation (Cho et al., 2002; Gebel et al., 2010). To study the protective mechanism of ILG on CSinduced COPD, the expression of NF- $\kappa \mathrm{B}$ and Nrf2 were tested in the present study. The data showed that CS exposure activated NF- $\kappa \mathrm{B}$ and Nrf2 signaling pathways in the lung. And these changes were inhibited by ILG treatment. The above results suggested that ILG inhibited CS-induced COPD through

\section{REFERENCES}

Chen, L., Sun, B. B., Wang, T., Wang, X., Li, J. Q., Wang, H. X., et al. (2010). Cigarette smoke enhances $\beta$-defensin 2 expression in rat airways via nuclear factor-кB activation. Eur. Respir. J. 36, 638-645. doi: 10.1183/09031936. 00029409

Chen, X., Cai, X. D., Le, R. R., Zhang, M., Gu, X. M., Shen, F. X., et al. (2018). Isoliquiritigenin protects against sepsis-induced lung and liver injury by reducing inflammatory responses. Biochem. Biophys. Res. Commun. 496, 245-252. doi: 10.1016/j.bbrc.2017.11.159

Cheng, L., Li, F., Ma, R., and Hu, X. (2015). Forsythiaside inhibits cigarette smokeinduced lung inflammation by activation of Nrf2 and inhibition of NF-kappaB. Int. Immunopharmacol. 28, 494-499. doi: 10.1016/j.intimp.2015.07.011

Cho, H. Y., Jedlicka, A. E., Reddy, S. P., Kensler, T. W., Yamamoto, M., Zhang, L. Y., et al. (2002). Role of NRF2 in protection against hyperoxic lung injury in mice. Am. J. Respir. Cell Mol. Biol. 26, 175-182. doi: 10.1165/ajrcmb.26.2.4501

Churg, A., Cosio, M., and Wright, J. L. (2008). Mechanisms of cigarette smokeinduced COPD: insights from animal models. Am. J. Physiol. Lung. Cell Mol. Physiol. 294, L612-L631. doi: 10.1152/ajplung.00390.2007

Churg, A., Dai, J., Tai, H., Xie, C. S., and Wright, J. L. (2002). Tumor necrosis factor-alpha is central to acute cigarette smoke-induced inflammation and connective tissue breakdown. Am. J. Respir. Crit. Care Med. 166, 849-854. doi: 10.1164/rccm.200202-097OC

Du, Y., Ding, Y., Chen, X., Mei, Z., Ding, H., Wu, Y., et al. (2017). MicroRNA$181 \mathrm{c}$ inhibits cigarette smoke-induced chronic obstructive pulmonary disease by regulating CCN1 expression. Respir. Res. 18:155. doi: 10.1186/s12931-0170639-1

Eapen, M. S., Hansbro, P. M., Mcalinden, K., Kim, R. Y., Ward, C., Hackett, T. L., et al. (2017). Abnormal M1/M2 macrophage phenotype profiles in the small airway wall and lumen in smokers and chronic obstructive pulmonary disease (COPD). Sci. Rep. 7:13392. doi: 10.1038/s41598-017-13888-x

Forman, H. J., and Torres, M. (2002). Reactive oxygen species and cell signaling: respiratory burst in macrophage signaling. Am. J. Respir. Crit. Care Med. 166, S4-S8. doi: 10.1164/rccm.2206007

Gao, F., Zhang, J. M., Fu, C. M., Xie, X. M., Peng, F., You, J. S., et al. (2017). iRGDmodified lipid-polymer hybrid nanoparticles loaded with isoliquiritigenin to enhance anti-breast cancer effect and tumor-targeting ability. Int. J. Nanomed. 12, 4147-4162. doi: 10.2147/IJN.S134148

Gebel, S., Diehl, S., Pype, J., Friedrichs, B., Weiler, H., Schuller, J., et al. (2010). The transcriptome of $\mathrm{Nrf2}(-/-)$ mice provides evidence for impaired cell cycle progression in the development of cigarette smoke-induced emphysematous changes. Toxicol. Sci. 115, 238-252. doi: 10.1093/toxsci/kfq039

Hou, C. L., Li, W. G., Li, Z. Y., Gao, J., Chen, Z. J., Zhao, X. Q., et al. (2017). Synthetic isoliquiritigenin inhibits human tongue squamous carcinoma cells inhibiting inflammatory reaction and oxidative stress, which are involved in the regulation of the Nrf2 and NF-кB signaling pathway.

\section{CONCLUSION}

In conclusion, our results indicated that ILG significantly inhibited CS-induced COPD, and that the mechanism of this effect might involve regulation of the Nfr2 and NF- $\kappa \mathrm{B}$ signaling pathways.

\section{AUTHOR CONTRIBUTIONS}

DY, ZM, and TW designed the experiments. DY, XL, GZ, and ZM did the experiments. TW analyzed the data. DY and XL wrote the article.

through its antioxidant mechanism. Oxid. Med. Cell. Longev. 2017, 1379430. doi: $10.1155 / 2017 / 1379430$

Ji, B., Guo, W., Ma, H., Xu, B., Mu, W., Zhang, Z., et al. (2017). Isoliquiritigenin suppresses IL-1beta induced apoptosis and inflammation in chondrocyte-like ATDC5 cells by inhibiting NF-kappaB and exerts chondroprotective effects on a mouse model of anterior cruciate ligament transection. Int. J. Mol. Med. 40, 1709-1718. doi: 10.3892/ijmm.2017.3177

Li, C., Yan, Y., Shi, Q., Kong, Y., Gao, L., Bao, H., et al. (2017). Recuperating lung decoction attenuates inflammation and oxidation in cigarette smoke-induced COPD in rats via activation of ERK and Nrf2 pathways. Cell Biochem. Funct. 35, 278-286. doi: 10.1002/cbf.3273

Li, N., Yang, L. A., Deng, X. N., and Sun, Y. A. (2018). Effects of isoliquiritigenin on ovarian cancer cells. Onco Targets Ther. 11, 1633-1642. doi: 10.2147/OTT. S149295

Li, R., Wang, X., Qin, T., Qu, R., and Ma, S. (2016). Apigenin ameliorates chronic mild stress-induced depressive behavior by inhibiting interleukin-1beta production and NLRP3 inflammasome activation in the rat brain. Behav. Brain Res. 296, 318-325. doi: 10.1016/j.bbr.2015.09.031

Liu, Q., Lv, H., Wen, Z., Ci, X., and Peng, L. (2017). Isoliquiritigenin activates nuclear factor erythroid-2 related factor 2 to suppress the NOD-like receptor protein 3 inflammasome and inhibits the NF-kappaB pathway in macrophages and in acute lung injury. Front. Immunol. 8:1518. doi: 10.3389/fimmu.2017. 01518

Luo, F., Liu, J., Yan, T., and Miao, M. (2017). Salidroside alleviates cigarette smokeinduced COPD in mice. Biomed. Pharmacother. 86, 155-161. doi: 10.1016/j. biopha.2016.12.032

Lykkegaard, J., Depont Christensen, R., Davidsen, J. R., Stovring, H., Andersen, M., and Sondergaard, J. (2013). Trends in the lifetime risk of COPD exacerbation requiring hospitalisation. Eur. Respir. J. 42, 964-971. doi: 10.1183/09031936. 00129312

Menghini, L., Ferrante, C., Leporini, L., Recinella, L., Chiavaroli, A., Leone, S., et al. (2016). An hydroalcoholic chamomile extract modulates inflammatory and immune response in HT29 cells and isolated rat colon. Phytother. Res. 30, 1513-1518. doi: 10.1002/ptr.5655

Moffet, J. R., Mahadeo, K. M., Fitzgerald, J., Gertz, S., Hall, M., Loomis, A. L., et al. (2016). The impact of neutrophil engraftment on the survival of intubated pediatric HCT patients: a pediatric acute lung injury and sepsis network study. Biol. Blood Marrow Transplant. 22, S60-S61. doi: 10.1016/j.bbmt.2015.11.353

Na, A. Y., Yang, E. J., Jeon, J. M., Ki, S. H., Song, K. S., and Lee, S. (2018). Protective effect of isoliquiritigenin against ethanol-induced hepatic steatosis by regulating the SIRT1-AMPK pathway. Toxicol. Res. 34, 23-29. doi: 10.5487/TR.2018. 34.1.023

Rangasamy, T., Cho, C. Y., Thimmulappa, R. K., Zhen, L., Srisuma, S. S., Kensler, T. W., et al. (2004). Genetic ablation of Nrf2 enhances susceptibility to cigarette 
smoke-induced emphysema in mice. J. Clin. Invest. 114, 1248-1259. doi: 10. 1172/JCI200421146

Shen, L. L., Liu, Y. N., Shen, H. J., Wen, C., Jia, Y. L., Dong, X. W., et al. (2014). Inhalation of glycopyrronium inhibits cigarette smoke-induced acute lung inflammation in a murine model of COPD. Int. Immunopharmacol. 18, 358-364. doi: 10.1016/j.intimp.2013.12.021

Shin, N. R., Sung-Hyeuk, P., Je-Won, K., Tae-Yang, J., Hyeun-A, K., JongChoon, K., et al. (2016). Melatonin attenuates neutrophil inflammation and mucus secretion in cigarette smoke induced airway inflammation via the suppression of Erk-Sp1 signaling. Respirology 21, 190-190. doi: 10.1111/jpi. 12192

Singh, A., Ling, G., Suhasini, A. N., Zhang, P., Yamamoto, M., Navas-Acien, A., et al. (2009). Nrf2-dependent sulfiredoxin-1 expression protects against cigarette smoke-induced oxidative stress in lungs. Free Radic. Biol. Med. 46, 376-386. doi: 10.1016/j.freeradbiomed.2008.10.026

Thayaparan, D., Shen, P., Stampfli, M. R., and Morissette, M. C. (2016). Induction of pulmonary antibodies against oxidized lipids in mice exposed to cigarette smoke. Respir. Res. 17:97. doi: 10.1186/s12931-016-0416-6

Traboulsi, H., Cloutier, A., Boyapelly, K., Bonin, M. A., Marsault, E., Cantin, A. M., et al. (2015). The flavonoid isoliquiritigenin reduces lung inflammation and mouse morbidity during influenza virus infection. Antimicrob. Agents Chemother. 59, 6317-6327. doi: 10.1128/AAC.01098-15

van der Vaart, H., Postma, D. S., Timens, W., and Ten Hacken, N. H. (2004). Acute effects of cigarette smoke on inflammation and oxidative stress: a review. Thorax 59, 713-721. doi: 10.1136/thx.2003.012468

Vogelmeier, C. F., Criner, G. J., Martinez, F. J., Anzueto, A., Barnes, P. J., Bourbeau, J., et al. (2017a). Global strategy for the diagnosis, management, and prevention of chronic obstructive lung disease 2017 report: gold executive summary. Arch. Bronconeumol. 53, 128-149.

Vogelmeier, C. F., Criner, G. J., Martinez, F. J., Anzueto, A., Barnes, P. J., Bourbeau, J., et al. (2017b). Global strategy for the diagnosis, management, and prevention of chronic obstructive lung disease 2017 report: gold executive summary. Eur. Respir. J. 49, 1700214. doi: 10.1183/13993003.00214-2017

Watanabe, Y., Nagai, Y., Honda, H., Okamoto, N., Yamamoto, S., Hamashima, T., et al. (2016). Isoliquiritigenin attenuates adipose tissue inflammation in vitro and adipose tissue fibrosis through inhibition of innate immune responses in mice. Sci. Rep. 6:23097. doi: 10.1038/srep23097
Wu, C. H., Chen, H. Y., Wang, C. W., Shieh, T. M., Huang, T. C., Lin, L. C., et al. (2016). Isoliquiritigenin induces apoptosis and autophagy and inhibits endometrial cancer growth in mice. Oncotarget 7, 73432-73447. doi: 10.18632/ oncotarget.12369

Xu, Y. N., Meng, C., Liu, G. L., Yang, D., Fu, L. S., Zhang, M., et al. (2016). Classically activated macrophages protect against lipopolysaccharide-induced acute lung injury by expressing amphiregulin in mice. Anesthesiology 124, 1086-1099. doi: 10.1097/ALN.0000000000001026

Xueshibojie, L., Duo, Y., and Tiejun, W. (2016). Taraxasterol inhibits cigarette smoke-induced lung inflammation by inhibiting reactive oxygen speciesinduced TLR4 trafficking to lipid rafts. Eur. J. Pharmacol. 789, 301-307. doi: 10.1016/j.ejphar.2016.07.047

Zeng, J., Chen, Y. Z., Ding, R., Feng, L., Fu, Z. H., Yang, S., et al. (2017). Isoliquiritigenin alleviates early brain injury after experimental intracerebral hemorrhage via suppressing ROS- and/or NF-kappa B-mediated NLRP3 inflammasome activation by promoting Nrf2 antioxidant pathway. J. Neuroinflammation 14:119. doi: 10.1186/s12974-017-0895-5

Zheng, J. P., Kang, J., Huang, S. G., Chen, P., Yao, W. Z., Yang, L., et al. (2008). Effect of carbocisteine on acute exacerbation of chronic obstructive pulmonary disease (PEACE study): a randomised placebo-controlled study. Lancet 371, 2013-2018. doi: 10.1016/S0140-6736(08)60869-7

Zuo, L., He, F., Sergakis, G. G., Koozehchian, M. S., Stimpfl, J. N., Rong, Y., et al. (2014). Interrelated role of cigarette smoking, oxidative stress, and immune response in COPD and corresponding treatments. Am. J. Physiol. Lung. Cell. Mol. Physiol. 307, L205-L218. doi: 10.1152/ajplung.00330.2013

Conflict of Interest Statement: The authors declare that the research was conducted in the absence of any commercial or financial relationships that could be construed as a potential conflict of interest.

Copyright (c) 2018 Yu, Liu, Zhang, Ming and Wang. This is an open-access article distributed under the terms of the Creative Commons Attribution License (CC BY). The use, distribution or reproduction in other forums is permitted, provided the original author(s) and the copyright owner(s) are credited and that the original publication in this journal is cited, in accordance with accepted academic practice. No use, distribution or reproduction is permitted which does not comply with these terms. 